\title{
The Level of Alkaline Phosphatase in Saliva as Biomarker for Pubertal Growth Phase
}

\author{
Fadhlina Irham \\ Faculty of Dentistry, Universitas Sumatera Utara \\ Medan, Indonesia \\ ina_kirei@yahoo.com
}

\author{
Siti Bahirrah \\ Department of Orthodontic \\ Faculty of Dentistry, Universitas Sumatera Utara \\ Medan, Indonesia
}

\author{
Nazruddin \\ Department of Orthodontic \\ Faculty of Dentistry, Universitas Sumatera Utara \\ Medan, Indonesia
}

\begin{abstract}
The identification of the onset of pubertal growth phase in growing patients is very important for orthodontists to determine orthodontic treatment planning and timing. The timing of growth phase can be assessed using chronological age, skeletal age, physiological age, dental age and biomarkers. ALP is one of the biomarkers that can be easily obtained from saliva. The increase of ALP levels in growing subjects is one of the indications of bone mineralization. The objective of this study was to assess the level of ALP in growing subjects in relation to the pubertal growth phase and age. This research was an observational analytic research with cross sectional design. Samples in this study consisted of 57 healthy growing female subjects (age ranging from 8 to 15 years). Growth phase was assessed through the cervical vertebral maturation (CVM) method described by Hassel and Farman. The subjects were divided into three groups according to their growth phases, which were prepubertal (CVM 1 and CVM 2), pubertal (CVM 3 and CVM 4), and post pubertal (CVM 5 and CVM 6). Unstimulated whole saliva collected from each subject and the level of ALP were measured using spectrophotometer. Results showed that peak levels of ALP were observed in pubertal growth phase: $233.39 \pm 106.29$ (IU/L), followed by prepubertal growth phase: $192.87 \pm 69.02$ (IU/L), and post pubertal growth phase: 79.20 \pm 31.41 (IU/L). The highest ALP levels were found at 10-11 years, and at 8-9 years, 12-13 years, and 14-15 years respectively. As conclusion, the level of ALP in saliva can be used as biomarker for the pubertal growth phase identification to determine orthodontic treatment planning and timing.
\end{abstract}

Keywords-alkaline phosphatase (ALP), saliva, biomarker, cervical vertebral maturation (CVM), growth phase

\section{INTRODUCTION}

An understanding of growth events is important in the practice of clinical orthodontics. Maturation status has considerable influence on diagnosis, treatment goals, treatment planning, and the eventual outcome of orthodontic treatment $[1,2]$.

Growth modulation procedures, which bring about changes in the skeletal base such as the use of extra oral orthopedic forces or functional appliances, are based on active growth periods. These active growth periods have to be objectively assessed for both the timing and the amount of active growth vector or direction of growth. [1,2].

The determination of the active growth phase (growth spurt) can be assessed by using several growth indicators such as chronological age, skeletal age (skeletal maturation), physiological age (sexual maturation) and dental age. The chronological age is not reliable as variability is the rule of growth pattern. In most of the conditions, skeletal age is assessed to pinpoint identify the different phases of the growth spurt. Skeletal maturity assessment involves visual inspection of the developing bone and their initial appearance, sequential ossification and related changes in shape and size [1-3].

Skeletal maturity has been assessed by using different techniques, the classic method of assessing skeletal maturity is by using hand wrist radiographs and the cervical vertebral maturation (CVM) method [1-5]. However, bone growth and remodeling are not only under the control of local factors, but systemic factors also play a crucial role. New possibilities to predict the maturation stages might be provided by biomarkers, that avoid invasive X-ray exposure and they represent agents that are directly involved in bone growth and remodeling. The biomarkers were initially detected in serum, but nowadays they are also detected in saliva. Biomarkers in saliva and serum are: Insulin-like growth factor 1 (IGF-1), growth hormone $(\mathrm{GH})$, Creatinine and Alkaline phosphatase (ALP) [1,3,7,9].

The identification of biomarkers in saliva and its use as a diagnostic tool has many advantages. It is much easier to collect, sufficient quantities can be easily obtained for analysis and no specific laboratory devices are necessary. The collection of saliva is also far less invasive compared to other body fluids such as gingival crevicular fluid and serum $[6,9,10,11]$

The ALP is one of the biomarkers that can be easily obtained from saliva, this enzyme plays a role in bone mineralization, the role of ALP in mineralization is provided by studies of the phased expression of genes 
during osteoblastic differentiation and growth plate cartilage calcification. In both tissues, bone and calcifying cartilage, ALP is expressed early in development [12,13]. Perinetti, et al, have shown increase of ALP level in gingival crevicular fluid (GCF) during puberty [7]. Travade, et al, detected increase of salivary ALP level in growing children [6]. Turan et al detected increase of serum ALP levels at infancy and puberty [15].

This study aims to assess the ALP levels in growing female subjects in relation to the pubertal growth phases and age.

\section{MATERIALS AND METHODS}

This research was an observational analytic research with cross sectional design. Population of this research were female patients who came to clinic of the Orthodontic Department, Faculty of Dentistry University of Sumatera Utara-Indonesia. The sample in this study were whole saliva and lateral cephalograms of 57 growing female subjects and the sampling was done using consecutive sampling method. The subjects in this study were included according to the following criteria i.e. female aged 8 to 15 years old, normal growth and healthy individuals (height, weight and chronological age of subjects were compared to ideal height, weight and age charts based on WHO), absence of systemic disease (e.g. bone disease and growth abnormality), absence of gingivitis and periodontal disease, didn't consume any medicine at the time of saliva sampling and willingly to participate in the study by filling the informed consent. Ethical clearance was obtained from the Research Ethics Committees of Faculty of Medicine, University of Sumatera Utara.

Lateral cephalograms were taken in natural head position for each subject. Growth phase was assessed through the CVM on lateral cephalogram using the method described by Hassel and Farman. This method compared six stages (CVM 1 to CVM 6) cervical vertebral maturation. The subjects were divided into three groups according to their growth phases i.e. prepubertal (CVM 1 and CVM 2), pubertal (CVM 3 and CVM 4), and post-pubertal (CVM 5 and CVM 6). These assessment are only done by an operator.

Subsequently, unstimulated whole saliva was taken in the morning at 10 to 12 o'clock GMT+7 hours, and subjects didn't consume any food or drinks 1 hour before salivary taking. After saliva collected, the whole saliva was clarified by centrifugation for 2 minutes at 10.000 RPM to remove insoluble material by using centrifuge machine. The supernatants saliva were collected by pipette into eppendrof tubes and frozen at $80^{\circ} \mathrm{C}$ until biochemical analysis. The ALP assay in saliva was done by the use of commercial kit manufactured by BioAssay Systems-USA, thereafter saliva were analyzed by using spectrophotometer at 405 $\mathrm{nm}$ in room temperature.

Data were checked for assumptions of normality by Shapiro-Wilk test and Levene test. Since data follows normality, parametric tests were used. The ALP levels among groups were compared by mean using Oneway ANOVA. Intergroup differences between growth phases were tested by using LSD test. Data were analyzed by using SPSS for Windows (version 18.0).

\section{RESULTS}

Normality and homogeneity tests were performed on all data using Shapiro-Wilk test and Levene test and the results showed that the data came from the same variant. Because of the homogeneous data, ALP levels among growth phases is done by using oneway ANOVA test with the degree of significance $\alpha=0.05$.

Table I shows the Oneway ANOVA test results $(\alpha=$ 0.05), there were significant differences in ALP levels among the growth phases $(p=0.0001)$. The highest mean of ALP levels was observed in the pubertal growth phase with a mean value 233,39 $\pm 106,29 \mathrm{IU} / \mathrm{L}$, followed by prepubertal growth phase with a mean value $192,87 \pm 69,02 \mathrm{IU} / \mathrm{L}$ and the lowest mean of ALP was found observed in the post pubertal growth phase with a mean value 79,20 $\pm 31,41 \mathrm{IU} / \mathrm{L}$ (Figure I).

TABLE I. MEAN OF ALP LEVELS VALUES BY GROWTH PHASES

\begin{tabular}{|l|c|c|c|}
\hline Growth Phases & $\mathbf{N}$ & $\mathbf{X} \pm \mathbf{S D}(\mathbf{I U} / \mathbf{L})$ & $\mathbf{p}$ \\
\hline Prepubertal & 19 & $192,87 \pm 69,02^{*}$ & $0,0001^{\mathrm{a}}$ \\
\hline Pubertal & 19 & $233,39 \pm 106,29^{*}$ & $0,0001^{\mathrm{a}}$ \\
\hline Post pubertal & 19 & $79,20 \pm 31,41^{*}$ & $0,0001^{\mathrm{a}}$ \\
\hline
\end{tabular}

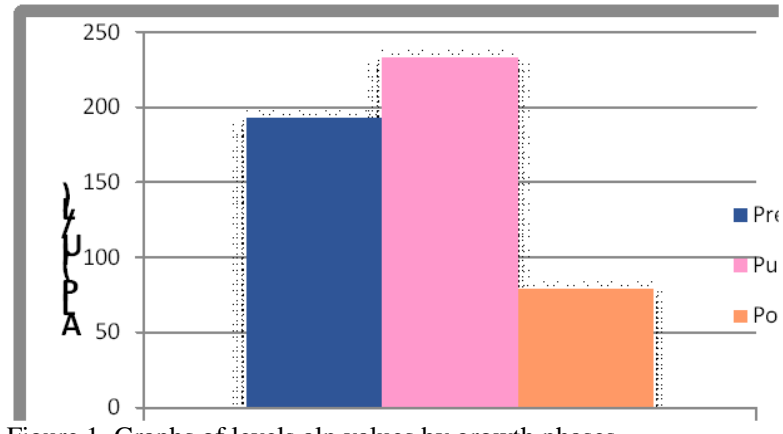

Figure 1. Graphs of levels alp values by growth phases.

Intergroup comparison was performed between different growth phases using LSD test, table II showed that there were significant difference $(p=0.0001)$ in ALP levels between post pubertal growth phase and prepubertal growth phase and between post pubertal growth phase and pubertal growth phase. Whereas between prepubertal and pubertal growth phase statistic results showed that there was no difference in ALP levels $(\mathrm{p}=0.103)$.

TABLE II. INTERGROUP DIFFERENCE OF ALP LEVELS VALUES BY GROWTH PHASES

\begin{tabular}{|c|c|c|c|}
\hline \multicolumn{2}{|c|}{ Growth Phases } & $\begin{array}{c}\text { Mean } \\
\text { Difference }\end{array}$ & p \\
\hline Prepubertal & Pubertal & $-40,52$ & 0,103 \\
& Post pubertal & 113,66 & $0,0001^{\mathrm{b}}$ \\
\hline Pubertal & Prepubertal & 40,52 & 0,103 \\
& Post pubertal & 154,19 & $0,0001^{\mathrm{b}}$ \\
\hline
\end{tabular}


Table III shows the Oneway ANOVA test results ( $\alpha$ $=0.05$ and $p=0.0001)$, the highest ALP levels were found at 10-11 years: $269,91 \pm 95 \mathrm{IU} / \mathrm{L}$, then $8-9$ years: $176,90 \pm 57,72 \mathrm{IU} / \mathrm{L}$, and then $12-13$ years: $134,40 \pm$ $83,31 \mathrm{IU} / \mathrm{L}$ and the lowest ALP levels at 14-15 years: $86,98 \pm 35,63 \mathrm{IU} / \mathrm{L}$ (Figure II).

TABLE III. MEAN OF ALP LEVELS BY AGE GROUPS

\begin{tabular}{|l|c|c|c|}
\hline Age (years) & $\mathbf{n}$ & $\mathbf{X} \pm \mathbf{S D}(\mathbf{I U} / \mathbf{L})$ & $\mathbf{P}$ \\
\hline $8-9$ & 10 & $176,90 \pm 57,72$ & $0,0001^{\mathrm{c}}$ \\
\hline $10-11$ & 14 & $269,91 \pm 95$ & $0,0001^{\mathrm{c}}$ \\
\hline $12-13$ & 25 & $134,40 \pm 83,31$ & $0,0001^{\mathrm{c}}$ \\
\hline $14-15$ & 8 & $86,98 \pm 35,63$ & $0,0001^{\mathrm{c}}$ \\
\hline
\end{tabular}

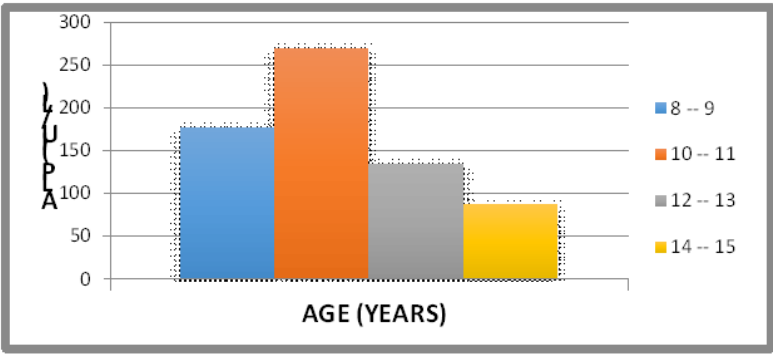

Figure 2. Graphs of APL level values by age groups.

Table IV shows intergroup comparison of ALP levels between different age groups using LSD test, there were highly significant differences $(p=0.0001)$ of ALP levels between 10-11 years and 12-13 years, also between 10-11 years and 14-15 years. Similarly, that were significant differences between age 8-9 years and $10-11$ years $(\mathrm{p}=0.006)$, between age $8-9$ years and 14 15 years $(\mathrm{p}=0.019)$.

TABLE IV. DIFFERENT SALIVARY CHARACTERISTIC IN SECC AND CARIES-FREE CHILDREN

\begin{tabular}{|c|c|c|c|}
\hline $\begin{array}{c}\text { Salivary } \\
\text { characteristic }\end{array}$ & SECC & Caries-free & P \\
\hline Flow rate $(\mathrm{ml} / \mathrm{min})$ & $0,37 \pm 0,12$ & $0.80 \pm 0.21$ & $<0.001^{\mathrm{d}}$ \\
\hline Salivary $\mathrm{pH}$ & $5,86 \pm 0,51$ & $7.02 \pm 0.39$ & $<0.001^{\mathrm{d}}$ \\
\hline Buffer Saliva & $4,93 \pm 1,38$ & $9.30 \pm 1.23$ & $<0.001^{\mathrm{d}}$ \\
\hline \multicolumn{4}{|c}{${ }^{\text {d. }}$ Unpaired t-test }
\end{tabular}

\section{DISCUSSION}

This study was done to assess ALP levels in 57 growing female subjects. The subjects in this study are divided into three groups i.e. the prepubertal group, the subjects categorized in this group were growing female with cervical vertebral maturation stage at CVM 1 and CVM 2. Then, the pubertal group, the subjects categorized in this group are growing female at with cervical vertebral maturation stage at CVM 3 and CVM 4. The last group is the postpubertal group, the subjects categorized in this group are growing female with cervical vertebral maturation stage at CVM 5 and CVM 6.

Assessment of cervical vertebral maturation stages using method described by Hassel and Farman, this method is chosen because it is easy to do, the assessment is done by looking at the shape and concavities that occur in inferior body of CV2, CV3, and CV4 that seen in lateral cephalogram $[4,5]$.

In this study, saliva was used as the sample, because it is much easier to collect in large quantities and the collection of saliva is far less invasive compared to other body fluids such as gingival crevicular fluid and serum $[6,10,11]$. Passive drool method was used in saliva collection, because this method will obtain whole saliva without stimulation [16,17]. Time of saliva collection for all subjects was done in the morning between $10-12 \mathrm{am}$, because at this time saliva flow rate was increase and reaches its peak at noon, it is caused by the circadian rhythm of the human body [18].

The level of ALP was measured by using colorimetric method (spectrophotometrically test) because, this test has the advantages of i.e. cheaper cost (compared with ELISA test), high sensitivity, wide range of ALP levels reading (ranging from 2-800 IU/L), easy and homogeneous procedure, and reliable $[15,19,20]$.

The Oneway ANOVA test in table I has responded to the hypothesis of the study, that there is a relationship between ALP levels in saliva and pubertal growth phases with mean difference in each group significant at $\mathrm{p}$ value 0.0001 . The highest ALP levels were seen at the pubertal phase (233.39 IU/L), then the prepubertal phase (192.87 IU/L), whereas the lowest ALP levels was seen in the post pubertal phase (79.20 IU/L). The results of this study were in accordance with a study conducted by Travade et al, which found an association between salivary ALP levels to middle phalanx of third finger (MP3) [6]. This present study is consistent with the study by Perinetti et al, which found a relationship between ALP levels in gingival crevicular fluid (GCF) in the maxilla, where the highest ALP levels occurred at pubertal stage $(78.8 \mathrm{IU} / \mathrm{L})$, then prepubertal stage (48.9 IU/L) and post-pubertal stage (21.9 IU/L) [14].

The LSD test in table II shows that there is a difference in ALP levels, which in the prepubertal phase is significantly different from the post pubertal phase, as well as the ALP levels at the pubertal phase which differ significantly from the post pubertal phase. This difference occurs due to changes in ALP levels at the time of bone mineralization during the growth period that begins to increase after 9 years old and decrease after 12 years old. 15

Traditionally, total serum ALP activity has been used as a biochemical marker for bone formation to assess osteoblastic activity in primary hyperparathyroidism, rickets, osteomalacia and Paget's disease. Since ALP is a marker for osteoblastic activity, growing children have higher levels than fully grown individuals. Highest levels of ALP are detected during the rapid growth phases of childhood such as infancy and puberty [15].

The Oneway ANOVA test in table III shows that in this study the highest ALP levels were seen at 10-11 years $(269.91 \mathrm{IU} / \mathrm{L})$, then at $8-9$ years $(176.90 \mathrm{IU} / \mathrm{L})$, at 12-13 years (134.40 IU/L), and the lowest ALP levels 
in the age range 14-15 years (86.98 IU/L). This is consistent with the study of Turan et al, which was found that the highest ALP activity in serum in female and male was found at 0-17 years, where the highest ALP levels in women occurred in the age range 10-11 years i.e. 587 (IU/L), then at 8-9 years 530 (IU/L), at $12-13$ years 515 (IU/L) and in the age range $14-15$ years decreased to 282 (IU/L).

Turan et al detected that the highest serum of ALP levels occurred during the first 6 months of life, then gradually diminished and began to rebound after 9 years old. ALP levels are almost the same between female and male up to age 10, but the highest ALP levels in female occur at age 10-11 years, and decreases after 12 years old. At 16-18 years old, levels of ALP in growing female will be similar to ALP levels in adult female [15].

As the conclusion, the results of this study may be useful in diagnosis and treatment plan by considering the level of ALP in saliva as biomarkers for the pubertal growth phase identification to determine orthodontic treatment planning and timing, so that orthodontists can determine the right time to perform orthopedic functional treatment and initiate orthognathic surgery.

\section{REFERENCES}

[1] S.M. Travade, S. Ramkrishna, "Skeletal maturity indicators," J Orthod. Res., vol. 3, pp. 158-161, March 2015.

[2] S.I. Bhalajhi, Orthodontics: the art and science, $5^{\text {th }}$ ed., New delhi: SK Arya, 2013, pp. 9-13, 213-236.

[3] S. Dhiman, S. Maheswari, S.K. Verma, "Assessment of maturity in orthodontics: A review," J. of Ad. Clin. Res. Insights, vol. 2, pp. 100-103, 2015.

[4] B. Hassel, A.G. Farman, "Skeletal maturation evaluation using cervical vertebrae," Am. J. Orthod. Dentofacial Orthop.., vol. 107, pp. 58-66, January 1995.

[5] S. Mahajan, "Evaluation of skeletal maturation by comparing the hand wrist radiograph and cervical vertebrae as seen in lateral cephalogram," Indian J. of Den. Res., vol. 22, pp. 309316, February 2011.

[6] S.M. Travade, S. Ramkrishna, S. Sarode, "Salivary alkaline phosphatase-a biochemical marker for growth prediction,"
Indian J. Basic and App. Med. Res., vol. 4, pp. 17-22, March 2015 .

[7] G. Perinetti, L. Franchi, A. Castaldo, L. Contardo, "Gingival crevicular fluid content and alkaline phosphatase activity in relation to pubertal growth phase, "Angle Orthodontist, vol. 82, pp. 1047-1052, June 2012.

[8] H. Wijaya, S. Redjeki, "Potential usage of salivary biomarkers as predictor for skeletal maturation in pubertal growth spurt," Proceeding book of Regional Oral Biology Scientific Meeting. Jakarta: Regional Oral Biology Scientific Meeting, pp. 38-45, 2014.

[9] S. Chiappin, G. Antonelli, R. Gatti, E.F. de Palo EF, "Saliva specimen: a new laboratory tool for diagnostic and basic investigation," Clinica Chimica Acta, vol. 383, pp. 30-40, 2007.

[10] R. Kumar, G. Sharma, "Salivary alkaline phosphatase level as diagnostic marker for periodontal disease," J. Int. Oral Health, vol. 3, pp. 81-85, May 2011.

[11] S.A. Ameer, A.F. Alhuwaizi, "The effect of orthodontic force on salivary levels of alkaline phosphatase enzyme," J. Bagh College Dentistry, vol. 27, pp. 175-179, December 2015.

[12] E.E. Golub, K. Boesze-Battaglia, "The Role of alkaline phosphatase in mineralization. Curr. Opin. Orthop., vol. 18, pp. 444-448, 2007.

[13] H. Orimo, "The mechanism of mineralization and the role of alkaline phosphatase in health and disease," J. Nippon Med. Sch., vol. 77, pp. 4-12, January 2010.

[14] G. Perinetti, L. Contrado, R. Di Lenarda, "Gingival crevicular fluid alkaline phosphatase activity as a non-invasive biomarker of skeletal maturation," Orthod. Craniofacial Res., vol. 14, pp. 44-50, 2011

[15] S. Turan, B. Topcu, I. Gokce, T. Guran, Z. Atay, et al., "Serum alkaline phosphatase levels in healthy children and evaluation of alkaline phosphatase z-scores in different types of rickets," J. Clin. Res. Ped. Endo, vol. 3, pp. 7-11, January 2011

[16] M. Navazesh, S.K. Kumar, "Measuring salivary flow; challenges and oppurtunities," J. Am. Dent. Assoc., vol. 139, pp 35-40, May 2008.

[17] D.T. Wong, Salivary diagnostic, Iowa: Wiley-Blackwell, 2008 pp. $37-42$.

[18] M. Hurlbutt, B. Novy, D. Young, "Dental caries: a pH-mediated disease," CDHA Journal, vol. 25, pp. 9-15, 2010.

[19] A.S. Kanagasabapathy, S. Kumari, Guidelines on standard operating procedures for clinical chemistry. New Delhi: Worl Health Organization, 2000, pp. 55-59.

[20] Anonymous, "Alkaline Phosphatase Assay Kit (DALP-250) Colorimetric Kinetic Determination of Serum Alkaline Phosphatase Activity," QuantiChrom: Bio Assay System, pp.1, 2009. 